6. Mortez, W.H. The anterior compartment ischemia syndrome. Am Surg 1953, 19: 729.

7. Whitesides, T.E., Jr, Hirada, H. \& Marimoto. K. Compartment syndromes and the role of fasciotomy. Its parameters and techniques. Am Acad Orthop Surg Instructional Course Lectures 1977, 26: 179-194.

8. Parving, H., Hansen, J.M., Nilsen, S.V., Rossing, N., Munck, O. \& Lassen, N.A. Mechanisms of edema formation in myxedema - increased protein extra-vasation and relatively slow lymphatic drainage. $N$ Engl J Med 1981, 301: 460.

9. Naeye, R.L. Capillary and venous lesions in myxedema. Lab Invest 1963, 12: 465-470.

10. Freedman, B.J. \& Knowles, C.H.R. Anterior tibial syndrome due to arterial embolism and thrombosis. Br Med J 1959, 2: 270.
11. Ramsay, I.D. Endocrine myopathies. Practitioner 1982, 226: 1075-1080.

12. Klein, I., Parker, M., Shebert, R. et al. Hypothyroidism presenting as muscle stiffness and pseudo-hypertrophy: Hoffman's syndrome. Am J Med 1981, 70: 891-894.

13. Pencea, V., Cotrutz, C., Dobrescu, G., Galasuna, C., Pencea, I.I. \& Dudeanu, I. Histopathological data on six cases of hypothyroidism accompanying muscular chronic disease. Rev Roumanie Med Endrocrinol 1985, 23: 105-109.

14. Bland, J.H. \& Frymoyer, J.W. Rheumatic syndromes of myxedema. $N$ Engl J Med 1970, 282: 1171-1174.

15. Garfin, S.R., Mubarak, S.J. \& Evans, K.L. Quantification of intra compartmental pressure and volume under plaster casts. J Bone Joint Surg 1981, 63A: 449-453.

\title{
Carcinoma of the breast at the site of migrated pacemaker generators
}

\author{
D.S. Bhandarkar, A.D.M. Bewu' and T.V. Taylor \\ Departments of Surgery and ${ }^{1}$ Cardiology, Manchester Royal Infirmary, Oxford Road, Manchester \\ $M 139 W L, U K$
}

\begin{abstract}
Summary: We report two patients in whom breast malignancy developed in the proximity of 'migrated' pacemaker generators. These cases and the similar ones reported in the literature raise concerns whether this association is merely coincidental or whether the pacemaker generator is responsible for the occurrence in some inexplicable manner. We urge for a routine, careful examination in all patients with implanted pacemaker generators at follow-up visits. This would help in (a) timely detection of migration of the pacemaker generators and (b) earlier diagnosis of any mass developing close to the migrated generators.
\end{abstract}

\section{Introduction}

We report, in two women, the development of breast cancer in the proximity of pacemaker generators, a rare association. Both the generators had migrated to a sub-mammary position from their original sites of implantation.

\section{Case reports}

Case 1

During a follow-up visit to the pacemaker clinic an 83 year old woman was detected to have a lump in

Correspondence: T.V. Taylor, M.D., Ch.M., F.R.C.S. (Eng.), F.R.C.S.(Ed.)

Accepted: 16 March 1993 her left breast. Thirteen years previously she underwent implantation of a Cordis Omni pacemaker to treat symptomatic sinus bradycardia and cardiac pauses. The pacemaker generator was changed to a Cordis 337A VVI type 6 years later. On both these occasions the generators were placed in left infraclavicular pockets. The lump noticed was situated in the outer, lower quadrant, was firm, ill-defined and free from the underlying structures. The overlying skin was tethered to it. The axillary or supraclavicular nodes were not palpable and the abdominal examination was unremarkable. Serum biochemical parameters were within normal limits. A plain X-ray of the chest (Figure 1) showed the pacemaker generator to be lying over the ribcage on the left side. This site corresponded with the clinically palpable lump. A fine-needle aspirate diagnosed it to be an adenocarcinoma. In view of 


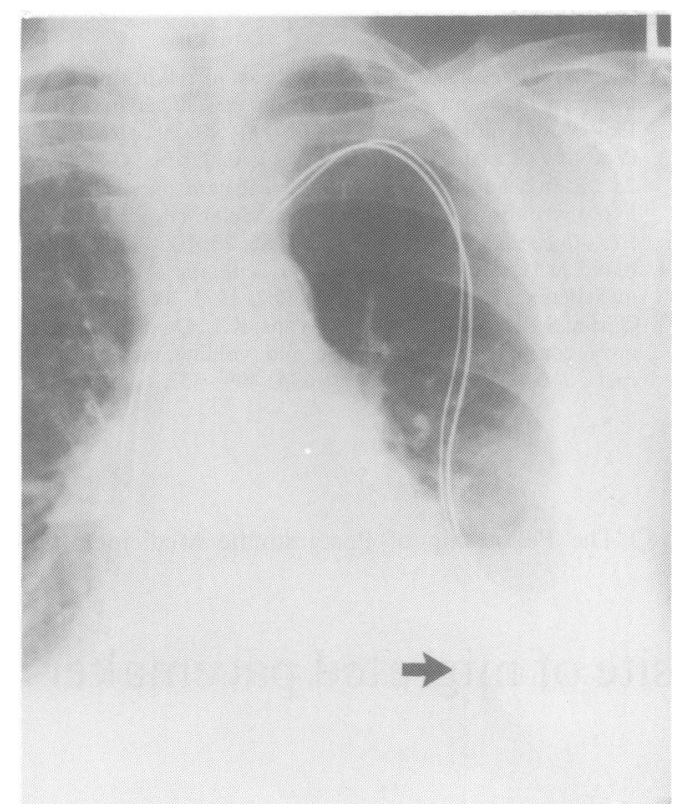

Figure 1 Arrow $(\rightarrow)$ points to the migrated pacemarker generator (Case 1).

her age, the patient was treated with anti-oestrogen therapy in the form of tamoxifen.

\section{Case 2}

An 84 year old woman was found to have a hard mass in the right breast at a follow-up visit to the pacemaker clinic. Her original pacemaker generator (Teletronics, VVI model 120B) had been inserted over the right precordium 12 years ago for sinus pauses. The system was replaced 10 years later with a Optima MP 580 VVI generator. The lump palpable was situated directly overlying the pacemaker generator which had migrated to an infra-mammary position. A fine-needle aspirate showed it to be an adenocarcinoma. In view of her age and her general poor condition, the patient was treated with tamoxifen.

\section{Discussion}

Carcinoma of the breast occurring in relation to a pacemaker generator is rare, and only four similar cases have been reported. ${ }^{1-3}$ Zafiracopoulos, ${ }^{1}$ who reported the first two cases, raised a question whether the association between breast malignancy and pacemaker generator was merely coincidental or that the two were correlated. Hamaker ${ }^{4}$ who reported a case of a plasmacytoma occurring in a pacemaker pocket proposed three possible explanations for the occurrence. He suggested that either: (a) the titanium cover of the generator was tumorogenic; (b) the electrochemical stimulation was responsible; or (c) it was due to inflammatory oncotaxis, ${ }^{5}$ a phenomenon in which chronically inflamed tissues or those lying close to a focus of constant irritation facilitate and trap tumour cells.

Our patients carried their initial pacemakers in the infraclavicular pocket and over the precordium, respectively, and presumably the changed generators also remained in situ for a few years. Later a malignancy was observed to develop in close proximity to each of the generators; both having migrated under the breast. In the two cases reported by Zafiracopoulos ${ }^{1}$ the generators had been placed 'under the breast', as indeed was the practice in the past. A woman ${ }^{2}$ in whom a pacemaker generator had been implanted over the right pectoral region was found to have an infiltrating breast carcinoma adjacent to it. She underwent a simple mastectomy. Two years later, with the generator still in place, a recurrence developed at the site of previous operation. In the only man reported to have developed breast malignancy im relation to a pacemaker generator, the generatoo had migrated to beneath the nipple. ${ }^{3}$

In view of the relatively high incidence of breast cancer, some tumours would be expected to develop in women in whom a pacemaker had been inserted. All the above mentioned cases have in common the placement of a pacemaker generator under the breast, by choice or by chance, and development of an adjacent malignancy. A pacemaker generator lying close could possibly be responsible for enhancing, by some ill-understood mechanism, the inherent potential of the breast tissue to develop malignancy. We urge the physicians involved in the care of patients with implanted pacemakers to be aware of this rare association. In these patients routine and careful examination at follow-up visits would allow (a) timely detection of migration of the pacemaker generators and (b) earlier diagnosis of a suspicious mass developing in the breast close to such migrated generators.

\section{Acknowledgement}

The authors wish to thank Ros Hirst, Chief Pacemaker Technician at the Manchester Royal Infirmary, for her help. 
1. Zafiracopoulos, P. Breast cancer at the site of implantation of pacemaker generator. Lancet 1974, 1: 1174.

2. Magilian, D.J. \& Isshak, G. Carcinoma of the breast in a pacemaker pocket - simple recurrence or oncotaxis? $P A C E$ 1980, 3: $220-223$.

3. Rasmussen, K., Grimsgaard, C. Vik-mo, H. et al. Male breast cancer from pacemaker pocket. PACE 1985, 8: 761-763.
4. Hamaker, W.R. \& Lindell, M.E. Plasmacytoma arising in a pacemaker pocket. Ann Thorac Surg 1976, 21: 354-356.

5. Der Hagopian, R.P., Sugarbaker, E.V. \& Ketcham, A. Inflammatory oncotaxis. $J A M A$ 1978, 240: 374-375.

\title{
First use of G-CSF in chlorpromazine-induced agranulocytosis: a report of two cases
}

\author{
J.R. Kendra, F.P. Rugman', T.A. Flaherty' ${ }^{1}$, A. Myers², N. Horsfield², \\ A. Barton ${ }^{2}$ and L. Russell ${ }^{3}$
}

Burnley General Hospital, Burnley, ${ }^{1}$ Preston Royal Infirmary, Preston, ${ }^{2}$ Blackburn Royal Infirmary, Blackburn and ${ }^{3}$ Amgen (UK) Limited, 240 Cambridge Science Park, Milton Road, Cambridge CB4 4WD, UK

\begin{abstract}
Summary: Chlorpromazine-induced agranulocytosis is an uncommon disorder associated with a high frequency of fatality. We describe two patients with chlorpromazine-induced granulocytosis in whom granulocyte colony stimulating factor (G-CSF) administration enhanced the speed of neutrophil recovery. No toxicity was noted with G-CSF and both patients made a successful recovery. We propose there is a role for such cytokine therapy in patients with life-threatening agranulocytosis in order to speed the recovery of neutrophils.
\end{abstract}

\section{Introduction}

Idiosyncratic non-cytotoxic drug-induced agranulocytosis is an unpredictable adverse reaction to a variety of drugs in hypersensitive individuals. Chlorpromazine-induced agranulocytosis is associated with a reported $50 \%$ mortality rate. ${ }^{1}$ The period of agranulocytosis may be extensive and during this period the patient is at considerable risk of developing serious infections requiring prolonged hospitalization. ${ }^{2}$

Neutrophil production is under the control of several glycoproteins including granulocyte colony stimulating factor (G-CSF). Clinical trials have demonstrated that treatment with G-CSF reduces the duration and incidence of agranulocytosis with fever in patients who have received cytotoxic chemotherapy. ${ }^{3}$ Furthermore the use of G-CSF is a proven benefit in the treatment of congenital or

Correspondence: J.R. Kendra, F.R.C.Path., Burnley Health Care NHS Trust, Burnley General Hospital, Casterton Avenue, Burnley, Lancs BB10 2PQ, UK. Accepted: 14 April 1993 cyclic agranulocytosis and neutrophils obtained from individuals treated with G-CSF function normally in vitro and in vivo. ${ }^{4,5}$

We report two cases of chlorpromazine-induced agranulocytosis which were reversed following therapy with G-CSF.

\section{Case reports}

Case 1

A 35 year old female with a long history of depressive illness was admitted 3 weeks after commencing chlorpromazine $40 \mathrm{mg}$ daily and procyclidine $5 \mathrm{mg}$ daily with a fever of $39^{\circ} \mathrm{C}$ associated with a sore throat.

Physical examination revealed aphthous ulceration of the buccal mucosa and an inflamed throat. A blood count showed a haemoglobin of $10.4 \mathrm{~g} / \mathrm{dl}$, a platelet count of $204 \times 10^{9} / 1$ and a white blood count of $0.3 \times 10^{9} / 1$. On the blood film only lymphocytes were detected. A bone marrow aspirate 